(Heidelberg) verliehen; die Laudatio wurde von Karl-Siegbert Rehberg (Dresden) gehalten.

\section{Aufbaustudium Kriminologie an der Universität Hamburg 2001}

Im Sommersemester 2001 beginnt der nächste Durchgang des viersemestrigen Aufbaustudiums Kriminologie (Abschluss: „Diplom-Kriminologe/ in").

Zulassungsvoraussetzungen: Abgeschlossenes Hochschulstudium in Soziologie, Psychologie, Pädagogik, Rechtswissenschaft oder in einem anderen der Kriminologie verwandten Fach (z.B. Sozialpädagogik, Politologie, Geschichte, Medizin) und Schwerpunktsetzung des bisherigen Studiums auf kriminologische Problemfelder.

Bewerbungsfrist: 15.12.2000 - 15.01.2001 (Ausschlussfrist!) beim Aufbau- und Kontaktstudium Kriminologie.

Kontakt: Prof. Dr. Sebastian Scheerer, Aufbauund Kontaktstudium Kriminologie, Troplowitzstraße 7, 22529 Hamburg, E-Mail: astksek@unihamburg.de; Internet: http://www.uni-hamburg. de/kriminol/
Stiftung Weltgesellschaft

Die Stiftung zur Förderung sozialwissenschaftlicher Forschung über die Weltgesellschaft - Stiftung Weltgesellschaft / World Society Foundation - fördert ausgewählte Forschungsprojekte zur Analyse der Weltgesellschaft und ihres Wandels.

Das Auswahlverfahren erfolgt neu ab 2001 zweistufig: Interessenten sind eingeladen, eine Skizze ihres Forschungsvorhabens von maximal 2 Seiten bis zum 31. März 2001 einzureichen. Forscher, deren Projektskizze positiv beurteilt wird, werden daraufhin eingeladen, ihr Forschungsprojekt bis zum 30 Juni auszuarbeiten. Die Finanzierung der defintiv bewilligten Forschungen kann im Januar 2002 anlaufen.

Kontakt: Stiftung Weltgesellschaft, c/o Soziologisches Institut der Universität Zürich; Rämistraße 69, CH-8001 Zürich; E-Mail: schindle@soziologie.unizh.ch; Internet: http://www.wsf.unizh.ch 